\title{
Merlionicity Part II: Familiarity Breeds Affection
}

\author{
Philip Hayward \\ University of Technology Sydney, and Southern Cross University, \\ Australia \\ prhshima@gmail.com \\ Publication Information: \\ Received 1 September 2017, Accepted 27 November 2017, Available online 19 January 2018 \\ doi: 10.21463/jmic.2017.06.2.06
}

\section{Abstract}

The merlion is a figure that comprises the upper half of a lion and the lower half of a fish. In an earlier issue of this journal (1[2], 2012), I charted the merlion's invention and introduction as a logo for Singaporean tourism in the 1960s and its memorialisation as a fountain statue in 1972. After examining a variety of cultural engagements with it during the 1970s-1990s, I concluded by analysing interpretations offered by artists contributing to the 2006 and 2011 Singaporean Biennales. In this follow-up article I explore the extent to which Singaporeans, expatriates and visitors have developed an increasing degree of affection for the figure. As subsequent sections detail, various recent deployments indicate that for some tourists, expatriates and Singaporean nationals, at least, the merlion is now regarded as a symbol that can be used in playful, ironic and/or often personalised manners, such as, most notably, a logo that they are willing to have inscribed on their flesh.

\section{Keywords}

Singapore, merlion, symbolism, affection, familiarity, tattoos

\section{Introduction}

On February 14th 2017 Singapore's Online Today newspaper published a report on a research project conducted on 35 Singaporean students to gauge their degree of "cultural attachment" to various national symbols (Yap and Christopoulus, 2017: online). The authors identified "cultural attachment" as concerned with "how we connect with others", and went on to assert that: 
just as children look to their caregiver as a secure attachment base [as] we grow, our need to bond directly with people is transferred indirectly to elements in our culture, whether it is a social group, values or cultural symbols. (ibid)

Contending that understanding the nature of cultural attachment is important "because it affects our reactions to threat and risk" (ibid), the research identified that the sample group rated the merlion most highly as a Singaporean symbol. The researchers also observed that it was "found to evoke a similar emotion that participants from the United States had for the Statue of Liberty and China [sic] students had for the terracotta warriors of Xi'an" (ibid). The research findings were notable for suggesting that a logo invented in 1962 (Fig. 1) that was initially viewed with considerable cynicism (at least from the educated populace) had established itself in the consciousness of younger generations as an engaging aspect of Singaporean national culture.

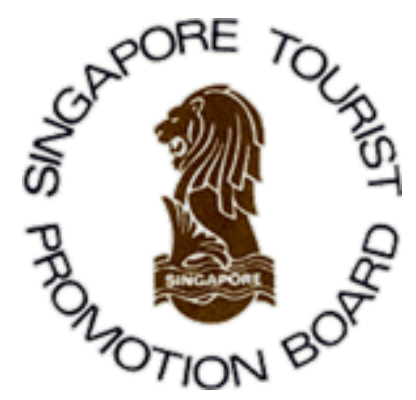

Fig 1. Original merlion logo design (1962)

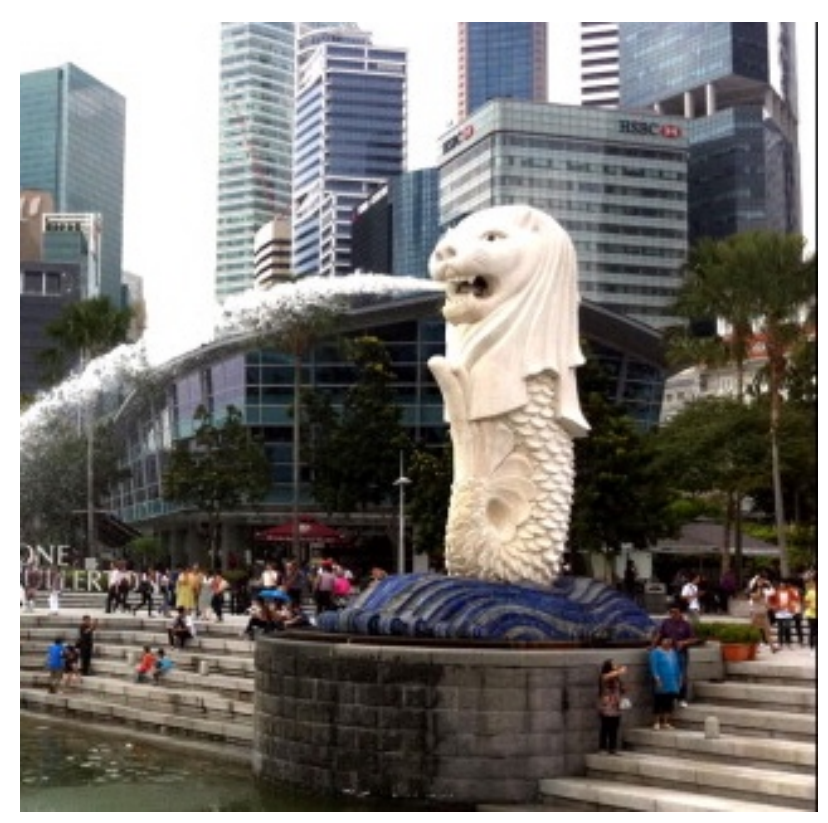

Fig 2. Merlion fountain statue (author's photograph, 2011) 


\section{Public Play}

In 2012 a celebration of the merlion's 40th anniversary was staged that included firework displays and light projections on and around the fountain statue (Fig. 2) and the screening of a video performance of a newly commissioned song of praise for it. ${ }^{1}$ In the light of the subtle and somewhat irreverent engagements with the merlion's public symbolism presented at the 2006 and 2011 Singapore Biennales (see Hayward, 2012), the 40th anniversary event seemed little more than a simplistic commemoration informed by somewhat dated notions of national populism. As if in implicit recognition of the latter, the celebration of Singapore's National Day (9th August) at Marina Bay in the following year was imbued with a more postmodern playfulness. The latter took the form of a video animation that was shown on a large screen in apparent interaction with performers located on a floating platform. As a group of singers attempted to perform a song, a loud male voice intruded into the sound mix. Apparently confused, the lead performer sought its source, only for the voice to ask them to look over to where he was. The source was then revealed on the screen as an animated merlion waving to them from a representation of the (actual) statue's location on the shore. Together with its friendly and humorous voice, the soft, fuzzy, luminousity of the animated figure (Fig. 3) gave it a far more inviting appearance than the inflexible form of its material statue. The latter aspect played a key role in the short video sequence that followed. After complaining about his immobility, which prevented him from joining in parade celebrations, the merlion gave a short, confused statement that satirised the very constructedness of his symbolism: "I have always wanted to take part in National Day ever since I was a young lion, a fish, errr mermaid, no... a mer-lion - Grrrr". After being invited to leave his pedestal and swim over to the platform (and being urged on by the crowd) he confessed that he was "a bit scared of water". When the lead singer voiced surprise, given that that he was "surrounded by water", he quipped back, "Why do you think I have never moved before? I'm half lion, lions don't like water." Cheered on to give him courage, ${ }^{2}$ the animated figure then dove off into the harbour and swam powerfully in a dolphin-like manner before mounting a surfboard (accompanied by a version of the theme tune to the US TV series Hawaii Five-O) before being dumped by a wave. He then swam underwater and danced together with sea creatures (accompanied by an instrumental version of the song 'Under The Sea', from Disney's 1989 film The Little Mermaid) before leaping up onto the performer's platform. Invited to tell the audience his story, the merlion then provided rap phrases to accompany the performance troupe's celebratory finale. ${ }^{3}$ While lighthearted, the brief video sequence both acknowledged the scrambled symbolism of a merlion - as the combination of an (aquaphobic) felid and a fish - and the fixity of its material form. Encouraged by a crowd of patriotic locals, the video animated merlion's leap from terrestrial Singapore into the aquatic space that surrounds the island can be understood as a joyous imaginative leap into a space very different to the polluted and crowded waterways that actually surround it. ${ }^{4}$ 


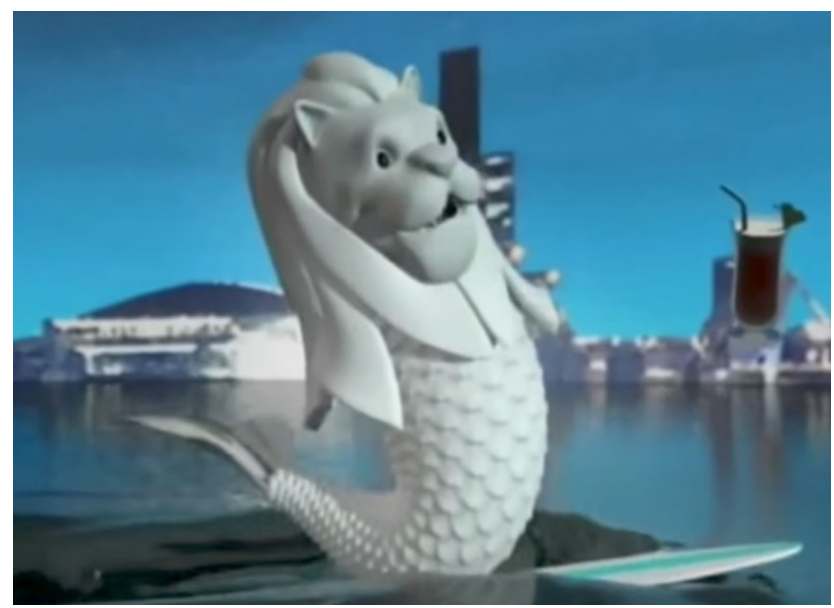

Fig 3. Surfing merlion, frame grab from National Day video presentation (2013)

Along with the officially facilitated public play discussed above, the merlion has also been the subject of crossassociation with another prominent international icon over the last decade - the Starbucks' coffee company logo. The cross association appears to derive from three main factors including:

1. the similarity of both designs in representing portmanteau fish/mammals (a mermaid in the case of the Starbucks' logo);

2. the prominence of both and the brand loyalty symbolised by and accruing to each; and, more specifically,

3. the location of a Starbucks' branch (and related signage) in Merlion Park, close to the fountain statue and its associated souvenir shop.

The Starbucks' logo has both similarities and differences to the merlion. Unlike the latter, the Starbucks logo was closely modeled on an earlier European medieval image - that of the split tailed mermaid. As detailed in Klara (2014), the Starbucks' logo - unlike the merlion - has undergone serial revision, effectively "zooming in" on the central area of earlier, full-body representations of the mermaid in order to arrive at the stable and now widely-known icon (represented at the lower left of Fig. 4). While not cited in any official company documentation, the apparent purpose of this was to remove any perception of sexual display that might have been conveyed by earlier images showing the figure holding her split tail apart (and potentially suggesting a genital area at the tails' point of convergence). The cross association of the merlion and Starbucks' logo has taken two main forms. One has occurred in official merchandising and, most particularly, in the form of the Singapore mug designs that were produced in 2015 as part of Starbucks' ongoing series of city souvenirs. As the promotional image for this product shows (Fig. 4) the separate Starbucks logo and postcard representation of the merlion fountain statue are linked by the design of the Singapore mug and are accompanied by a lower image caption that gives a condensed version of the official explanation for the merlion's design. ${ }^{5}$ This type of packaging and promotion represents a relatively uncomplicated cross-promotion of two symbols (and respective institutions) through which both gain prominence and traction. More irreverent, unauthorised cross associations have also occurred in recent years. ${ }^{6}$ The opportunistic shopping bag design (Fig. 5a), for instance, playfully merges the two icons in a design that is (unconcernedly) incoherent in its anatomy, featuring the curled frontal tail aspect of the merlion with the split tailed design of Starbucks' mermaid. While more anatomically coherent, The Guardian Angel company's 'Singapore Lion City' t-shirt (Fig. 5b) takes the playfulness a step further by virtue of being a novelty glow-in-the-dark 
product that evokes the Starbucks' logo through its circular design and use of green as a background colour, substituting the mermaid's face, hair and upper torso with that of a (friendly looking) merlion's head and the upper protrusion of its tail.

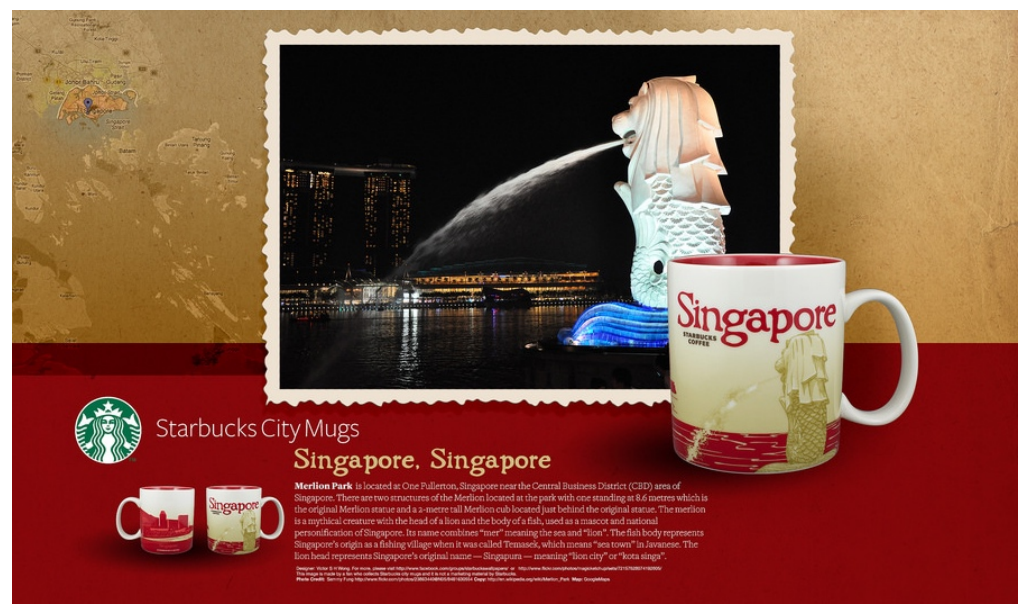

Fig 4. Starbucks promotional poster for their official Singapore city mug (2015)

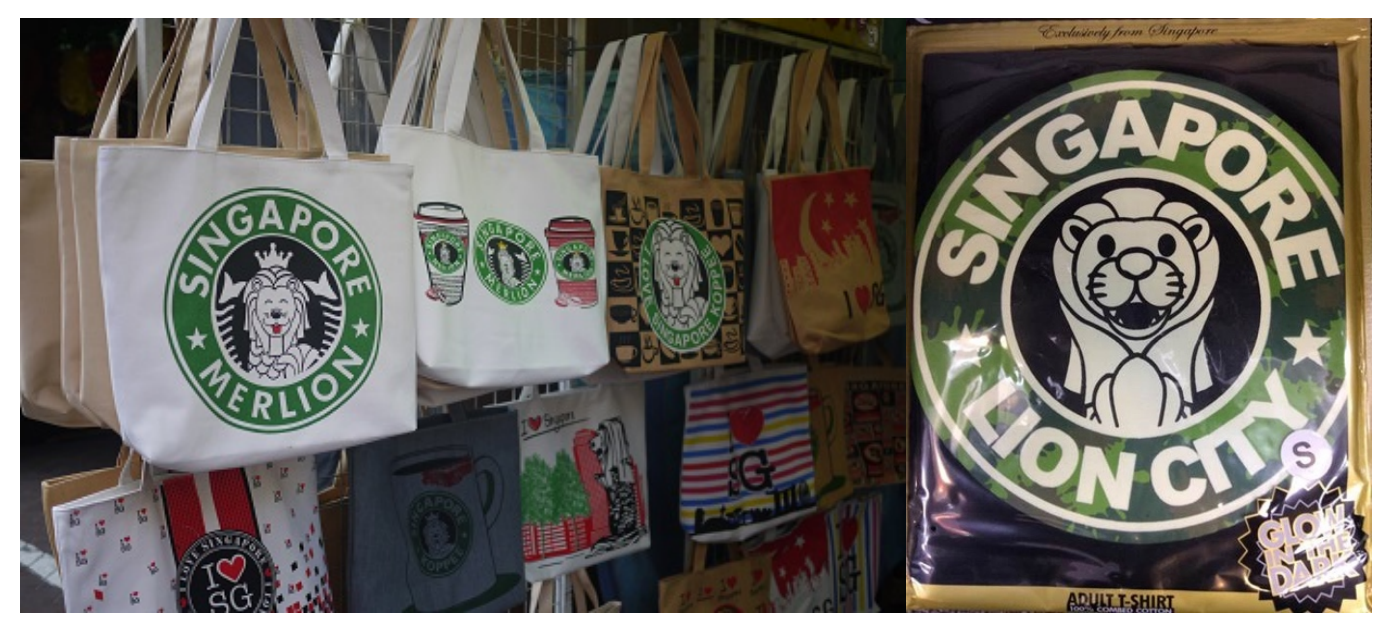

Fig 5. a) Canvas merlion bag (c2015, image by MISS HAPPY) and b) front image of novelty merlion t-shirt design (c2016)

\section{Body Play}

If the 2013 National Day video and the product designs discussed above reflected a growing public familiarity with and affection for the merlion, another more private manifestation of these sentiments has occurred in the form of body tattoos. This phenomenon reflects a wider wave of body tattooing internationally over the past two decades and the 
more recent expansion of the practice in Singapore (which, in itself, serves as one indicator of loosening social conservatism and senses of social propriety in the island nation).

Research conducted by contacting Singaporean tattooists and tattooed individuals and through online image searches has revealed three distinct chronological phases of merlion tattoo acquisition (at least as perceived and related by informants). In this, foreign tourists appear to have been the initiators and first recipients of merlion tattoos from the mid-2000s onwards, followed by expatriate residents and then by (either resident, émigré and/or re-visiting) Singaporean nationals. ${ }^{7}$ In this regard, the diffusion pattern of mermaid tattoos represents various stages in the successful promotion of the merlion as a symbol for Singapore. Initially designed as tourist trademark, the merlion has developed as a national icon that is sufficiently potent and evocative to have been acquired as a skin souvenir by tourists, as a marker of temporary residence and/or a memento of that by expatriates and, most recently, as a symbol of national pride acquired by Singaporean nationals.

My research into the design of merlion tattoos has revealed that most follow the vertical alignment of the classic design (as in Figs. 1 and 2 above). ${ }^{8}$ As would be expected, larger tattoos have been executed on backs, upper arms, thighs or calves and smaller ones on ankles, lower wrists or forearms. Feroze McLeod, of the Bada Bink Tattoo Firm, specialises in merlion designs and has produced a range of them for tourists, expats and native Singaporeans since doing his first one for an Australian tourist in 2011. Characterising the merlion as a national "mascot," he has identified the symbol's strength as the manner in which it "can be reinterpreted in many ways that it is interesting for me to explore" (personal communication, 21st August, 2017). As the tattoo reproduced below (Fig. 6) indicates, his work involves dynamic and richly coloured interpretations of the classic merlion and versions that modify the standard position of the merlion and represent it with other symbols. ${ }^{9}$

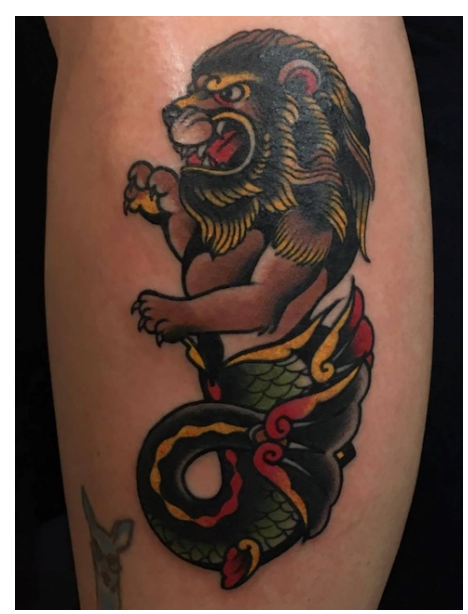

Fig 6. Merlion tattoo by Feroze McLeod (2015, design copyright: the artist)

Showing more humorous creative licence, another Singaporean tattooist, Aaron Tay, extended the parameters of the merlion design in 2012 by inking an overseas tourist with an orange headed lion mounted on a red prawn tail (an image Tay has called a "singa-prawn"). ${ }^{10}$ The temporary henna tattooists of Singapore's Little India Arcade have also included 
the merlion in their design repertoire in recent years, providing tourists with the local symbol along with more standard designs (Fig. 7).

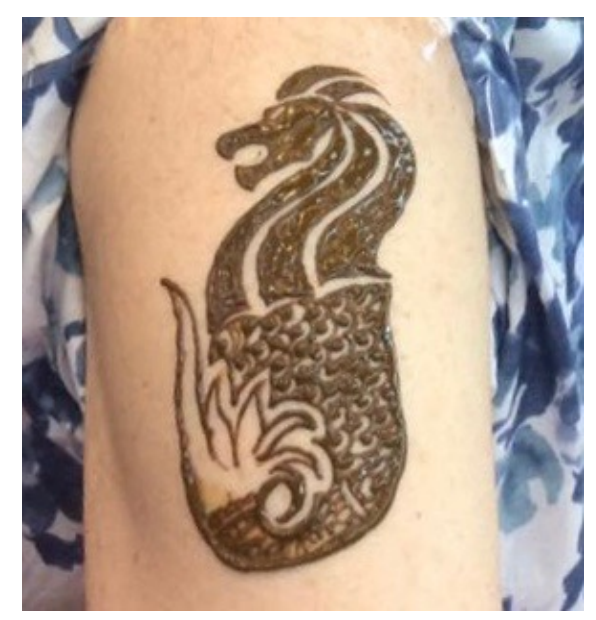

Fig 7. Merlion henna tattoo by Dipen (Little India Arcade) on the author's upper arm (2017)

While resident expatriates do not seem to have sought out such novelties as Tay's "singa-prawn," research indicates that some have chosen souvenir tattoos that combine the merlion with other iconic local images. One tattoo designed by LukaLit, for instance, featured the merlion and the triple pillared Marina Sands building superimposed over an outline of Singapore island. The artist subsequently described the work as "one of the most clichéd tatts of the year [but a fun challenge]". ${ }^{11}$ My research suggests that resident expats who acquire merlion tattoos after leaving Singapore tend towards smaller and less dramatic designs. Tiffany Hutton, an Australian national who recently acquired one such design (Fig. 8) has described her motivations for acquiring it in the following terms:

When I was growing up in Singapore in the 70s and 80s, the merlion was considered embarrassingly kitsch by everyone I knew. My parents were in advertising, so they were pretty cynical about it. But Singapore was an important part of my life - I was almost 17 when I left - and after a visit in February this year I decided to get a merlion tattoo on my ankle. I know some Singaporeans are disparaging about ang mos ${ }^{11}$ like myself getting merlion tattoos, but I like that I have a symbol of my childhood home, with all its contradictions and quirks, tattooed on me. (personal communication, August 27th 2017) 


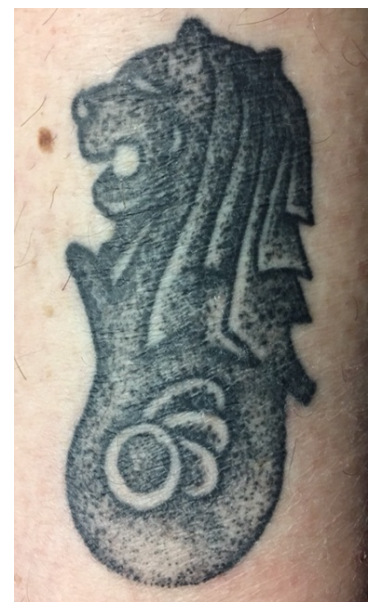

Fig 8. Merlion design by Australian tattooist Brett Eberhard on Tiffany Hutton (2017)

One of the first females to attract attention for having a merlion tattoo was Kaylani Lei, a Singaporean-born actress and model of Chinese and Filipino descent who has been active in the US porn industry since 2004. Video sequences and photographs of her taken around 2010 show a prominent merlion (in standard erect posture and with a green scaled tail) on the left side of her upper back (Fig. 9, left). In an item on the Singaporean Blog 'Alvinology' published in 2012, the blogger includes a photo of the tattoo and text that expresses his surprise at the image, stating that, "most of the Merlion Tattoos seem to be found on foreigners... I really doubt any Singaporeans would want a Merlion stamped anywhere on our bodies". A number of respondents countered this, expressing affection for the icon, making comments like "Actually, now that a casino is our national symbol, the merlion makes me pretty nostalgic" and suggesting that "an ironic hipster tattoo based on the original 1970s logo" would be appealing (ibid). Another poster commented that Lei's tattoo was "pretty cool" and added that, "at least she still helps to promote Singapore when she bares her back" (ibid). By 2014 images of the actress' back showed that the original merlion image had been augmented by decorative wing and tail designs (Fig. 9, right), making the merlion even more prominent.

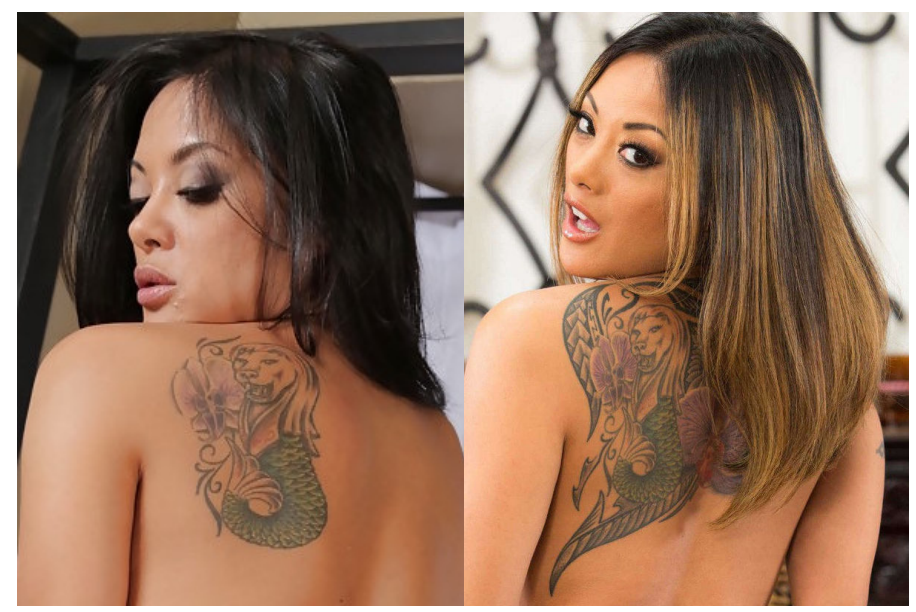

Fig 9. Kaylani Lei's merlion tattoo(s) (c2009 and c2014) 
While men appear to form the majority of the resident Singporean nationals who choose to acquire tattoos, there has been a significant development in recent years. Somewhat surprisingly, in a society where tattooing has traditionally been limited to particular male subcultures and where social conservatism and notions of propriety have militated against women having tattoos, there has been a recent rise in the number of Singaporean women being adorned. Commencing with the characterisation that "not so long ago" a body tattoo "was more likely to have its roots in gang affiliations, a prison term, or rebellion against conventional values," Tan (2017: online) profiled a number of professional women who have been tattooed and identified their motivation in terms of tattoos being "now part of the narrative of who you are, what you believe in, and what you cherish" (ibid). Female Singaporeans are also significant for being early national adopters of merlion tattoos, reinforcing the extent to which the mermaid now appeals to a wide sector of the population. One striking example occurs on the forearm of a young Singaporean female ${ }^{13}$ who has been featured on social media sites (Fig. 10). Together with her other tattoos, it presents a new and markedly different image of Singaporean femininity to the best-known icon of national womanhood - the immaculately groomed Singapore Airways Girl in her distinctive sarong kebaya. ${ }^{14}$

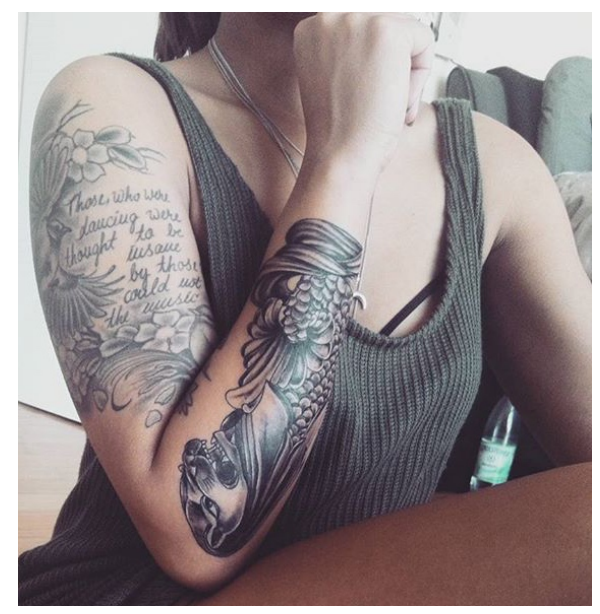

Fig 10. Merlion tattoo on forearm of young Singaporean woman $(2017)^{15}$

\section{Conclusion}

This short, follow-up essay to my previous (2012) study of the deployment of the merlion in late 20th and early 21st Century Singapore identifies and analyses a new phase in the icon's perception and circulation in the island-state and amongst an extended community of individuals who have varying degrees of identification and/or engagement with it. In this regard, the essay provides further evidence of the merlion's success as a symbol of Singapore and documents a shift in senses of familiarity and affection concerning it. Far from being the sterile and artificial corporate logo that its initial detractors identified, it is now a vibrant part of Singapore's vernacular identity and an international trademark that is beginning to approach the iconicity of New York's Statue of Liberty or - in terms of Singapore's increasingly corporatised culture - the ubiquitous Starbucks' logo. In this regard, the merlion's inscription on the flesh of tourists, 
expatriates and citizens can be considered as the latest and most successful indication of the manner in which it has come to represent the country.

\section{Acknowledgements}

Thanks to Amelia Coyle-Hayward for accompanying and assisting me on field research for this article.

\section{Endnotes}

1. See highlights of the event online at: https://www.youtube.com/watch?v=NHuxHtXQgA0 - accessed 28th March 2017.

2. This interaction evokes comparison to the encouragement of the faint-hearted lion in the 1940 Hollywood film The Wizard of Oz.

3. See a video recording of this sequence online at: https://www.youtube.com/watch?v=vmUl-59WZgM - accessed 28th March 2017.

4. Despite the Singaporean Government passing the 'Prevention of Pollution of the Sea Act' in 1990 (and subsequently enhancing and amending it), Singapore's waters remain heavily polluted.

5. Starbucks' social media eagerly covered the creation and local popularity of a Singaporean variant of the company's pink 'Unicorn' Frappucino drink (briefly available in the US in April 2017). Created by staff at the Sunset Point Starbucks café, and dubbed the 'Merlion Frappucino' in social media, the (brief) local vogue for the drink received mainstream media attention in Singapore (see, for instance Min, 2017: online).

6. Indeed, there has been a plethora of unauthorised modifications of the Starbucks logo internationally over the past two decades that merits research and critical attention for its variously critical, parodic and plagiaristic aspects.

7. Research also revealed merlion tattoos on foreign individuals with no apparent connections with Singapore who appear to have chosen the image on its own terms.

8. With some even representing the water spouts gushing from the statue's mouth - see, for example, Australian tattooist Dean Madden's design online at: http://www.thepicta.com/media/1488330667967916820_1293621358 - accessed 21st August 2017

9. Such as a 2016 design showing the merlion brandishing a trident of the sort commonly associated with Neptune and tritons in Western culture.

10. See image and comment online at: http://www.imgrum.org/media/807920267354609529_214035164 - accessed 21 st August 2017.

11. See image and comment online at: http://www.thepicta.com/media/983766949858605448_1797186501 - accessed 25th August 2017.

12. Ang mo - which literally means 'red haired' - is a mildly pejorative term used by native Singaporeans and Malaysians to refer to Caucasians. 
13. The individual's name and social media addresses have been withheld to provide anonymity.

14. See Lin (2015) for discussion.

15. As per footnote 13 above.

\section{References}

Alvinology, 2012. The Girl with the Merlion tattoo', Alvinology 4th November, online at: https://alvinology.com/2012/04/11/the-girl-with-themerlion-tattoo/

Hayward, P., 2012. Merlionicity: The twenty first century elaboration of a Singaporean symbol. Journal of Marine and Island Cultures 1(2). 113125.

Klara, R., 2014. How a Topless Mermaid Made the Starbucks Cup an Icon (She's a little more modest today than in the '70s). AdWeek September 29th, online at: http://www.adweek.com/news/advertising-branding/how-topless-mermaid-made-starbucks-cup-icon-160396

Lin, W., 2015. 'Cabin Pressure': designing affective atmospheres in airline travel. Transactions of the Institute of British Geographers 40(2). 287299.

Min, C. H., 2017. Canter over unicorn frappuccino, here's the Merlion frap from a Starbucks in Singapore. Straits Times 23rd April, online: http://www.straitstimes.com/lifestyle/food/canter-over-unicorn-frappuccino-heres-the-merlion-frap-from-a-starbucks-in-singapore

Tan, J., (2017) Ink-spiration: Why more and more Singaporean women are embracing tattoos. HerWorld Plus 15th May, online: http://www.herworldplus.com/lifestyle/tattoo-singapore-body-ink-bernice-chua-victoria-woon-jen-tan

Yap, W.J. and Christopoulos, G., 2017. The Merlion's power as a symbol for Singaporeans. Today Online 14th February, online: http://www.todayonline.com/commentary/merlions-power-symbol-singaporeans 